\title{
Biosafety and Toxicological Evaluation of Tissue-Cultured Echinacea purpurea Adventitious Roots
}

\author{
Hosakatte Niranjana Murthy ${ }^{1,2}$, So-Young Park ${ }^{1}$, Eun Jeong Lee ${ }^{1}$, and Kee Yoeup Paek ${ }^{1 *}$ \\ ${ }^{1}$ Research Center for the Development of Advanced Horticultural Technology, Chungbuk National University, Cheongju 361-763, Korea \\ ${ }^{2}$ Department of Botany, Karnatak University, Dharwad 580003, India
}

\begin{abstract}
Echinacea purpurea (L.) Moench (purple cone flower) is an important medicinal plant; it can enhance immunity, relieve pain, and reduce inflammation, and also has hormonal, antiviral, and antioxidant effects. Adventitious root biomass of Echinacea purpurea was produced in commercial-scale bioreactors for use as a dietary supplement in the food industry and in traditional medicine. Biosafety and toxicological evaluations of tissue-cultured Echinacea purpurea adventitious roots (TCEPARs) were performed. Reverse mutation and chromosomal aberration tests showed no significant mutagenicity. Furthermore, repeated four-week oral dose tests performed in Sprague-Dawley rats did not show any notable changes in the general behavior of the rats, in the gross appearance of their internal organs, or in their mortality rate. There were no differences between the control group and the treatment group in parameters such as absolute body weight, hematology, blood chemistry, and absolute and relative organ weights. These findings indicate that TCEPARs are safe and nontoxic when consumed at an average dietary level and can be used as raw material for traditional medicine and the food industry.
\end{abstract}

Additional key words: Echinacea, medicinal plant, mutagenicity, natural product, toxicology

\section{Introduction}

Echinacea purpurea (L.) Moench. (purple cone flower) is an important medicinal plant that is widely used in North America and Europe as an herbal drug for stimulating the immune system (e.g. for curing common cold), and as an antioxidant. The plant has various pharmacological properties such as antibacterial, antiviral, antifungal, and antitumor effects (Barrett, 2003; Miller, 2005). The most important potentially active compounds in Echinacea purpurea are caffeic acid derivatives, polysaccharides, alkamides, and glycoproteins (Barrett, 2003; Bauer and Wagner, 1991). Echinacea purpurea is used as a dietary supplement in the food industry, and studies show that the plant and its active components affect the phagocytic immune system (Percival, 2000). It is one of the top-selling herbal products in the world (Marriott, 2003; Wills et al., 2000).

The demand for Echinacea purpurea raw material and extract has increased in recent years (Blumenthal, 2003;
Yu and Kaarlas, 2004) but the production levels of the raw material produced through field cultivation are not adequate to meet the demand of the pharmaceutical and food industries. Furthermore, field cultivation affords a low yield and poor quality of raw material because of diseases that affect the plant and the natural variation in chemical composition caused by seasonal changes (Abbasi et al., 2007). However, in vitro culture techniques are proving very valuable for meeting the increasing demand of the pharmaceutical and food industries (Abbasi et al., 2007). Wu et al. (2007) produced adventitious root biomass of Echinacea purpurea through in vitro methods using commercial-scale bioreactors. Adventitious roots of Echinacea purpurea produced thusly have higher concentrations of bioactive compounds (Wu et al., 2007). Biosafety and toxicological evaluations of tissue-cultured adventitious roots are essential before marketing of the raw material for use by the pharmaceutical and food industries.

The objective of the study was to evaluate the toxicity

\footnotetext{
*Comesponding author: paekky@chungbuk.ac.kr

※ Received 8 June 2014; Revised 19 August 2014; Accepted 17 September 2014.

(C) 2015 Korean Society for Horticultural Science
} 
and safety of tissue-cultured Echinacea purpurea adventitious roots (TCEPARs) in view of a planned release as a traditional medicine or functional food supplement. TCEPARs can be used in traditional medicine, nutraceutical products, and functional foods with medical benefits. Therefore, due to their possible widespread use, a range of toxicological tests were performed to investigate their safety.

\section{Materials and Methods}

\section{Adventitious Root Production of Echinacea purpurea}

Adventitious roots of Echinacea purpurea were produced in $1000 \mathrm{~L}$ commercial scale bioreactors using the methods described by Wu et al. (2007). The adventitious root biomass was air dried using forced hot air drying methodology. The powdered material containing higher concentrations of bioactive compounds such as caftraic acid $\left(3.9 \mathrm{mg} \cdot \mathrm{g}^{-1}\right.$ DW), chlorogenic acid (4.9 mg. $\left.\mathrm{g}^{-1} \mathrm{DW}\right)$ and cichoric acid (22.5 $\mathrm{mg} \cdot \mathrm{g}^{-1} \mathrm{DW}$; Wu et al., 2007) was used for biosafety and toxicological analysis. The tissue cultured Echinacea purpurea adventitious root powder specifications are summarized in Table 1.

Table 1. Chemical analysis of tissue cultured Echinacea purpurea adventitious roots ${ }^{\mathrm{z}}$.

\begin{tabular}{lc}
\hline Parameters & Results \\
\hline Calories & $345 \mathrm{cal} / 100 \mathrm{~g}$ \\
Fat & $13 \mathrm{cal} / 100 \mathrm{~g}$ \\
Saturated fatty acid & $0.71 \mathrm{~g} \cdot 100 \mathrm{~g} \mathrm{~g}^{-1}$ \\
Cholesterol & $<1 \mathrm{mg} \cdot 100 \mathrm{~g}^{-1}$ \\
Carbohydrates & $57.9 \mathrm{~g} \cdot 100 \mathrm{~g} \mathrm{~g}^{-1}$ \\
Total dietary fiber & $28.1 \mathrm{~g} \cdot 100 \mathrm{~g} \mathrm{~g}^{-1}$ \\
Total sugars & $4.87 \mathrm{~g} \cdot 100 \mathrm{~g} \mathrm{~g}^{-1}$ \\
Moisture & $7.03 \mathrm{~g} \cdot 100 \mathrm{~g} \mathrm{~g}^{-1}$ \\
Total ash & $8.45 \mathrm{~g} \cdot 100 \mathrm{~g} \mathrm{~g}^{-1}$ \\
Protein & $25.2 \mathrm{~g} \cdot 100 \mathrm{~g} \mathrm{~g}^{-1}$ \\
Vitamin A & $<76 \mathrm{IU} / 100 \mathrm{~g}^{-1}$ \\
Vitamin C & $8.43 \mathrm{mg} \cdot 100 \mathrm{~g}^{-1}$ \\
Sodium & $7.84 \mathrm{mg} \cdot 100 \mathrm{~g}^{-1}$ \\
Calcium & $186 \mathrm{mg} \cdot 100 \mathrm{~g}^{-1}$ \\
Iron & $\mathrm{ND}$ \\
\hline ZAnlysi
\end{tabular}

${ }^{\mathrm{z}}$ Analysis was carried out by US FDA laboratory under the I. D. 2030513 dated 11/04/2007.

${ }^{\mathrm{y}}$ Not detected.

\section{Mutagenicity Test}

The Salmonella typhimurium strains TA98, TA100, TA1535 and TA1537 and the Escherichia coli strain WP2uvrA were used for the reverse mutation test, both with and without an S-9 mixture. Tests were carried out according to the method of Ames et al. (1975) using tissue cultured Echinacea purpurea adventitious root powder doses at 312.5, 625, 1250,2500 , and $5000 \mu \mathrm{g} /$ plate.

\section{In vitro chromosomal aberration test with Chinese Hamster} Lung cells: A chromosomal aberration test was performed using Chinese Hamster Lung (CHL) cultured cells (Dainippon Pharmaceutical Co., Ltd.) in both non-activated and activated system, with and without a S9 mixture, for $6 \mathrm{~h}$, and also in a non-activated system without a S9 mixture for 24 $\mathrm{h}$, at doses of 275, 550, $1100 \mu \mathrm{g} \cdot \mathrm{mL}^{-1}$. Mitomycin C (Sigma, USA) was used as a positive control.

Toxicologicalstudies: Four weeks repeated dose toxicity (oral) test was carried out in collaboration with drug testing laboratory Biotoxtech, South Korea on experimental Sprague Dawley rats. All the animals involved were maintained on a standard diet and kept in a room with environmentally controlled conditions of $24 \pm 1^{\circ} \mathrm{C}$ and $12 \mathrm{~h}$ light: $12 \mathrm{~h}$ dark cycles. The animals had free access to water and a standard diet [fed with a normal laboratory commercial stock diet containing $16 \%$ protein, $56 \%$ carbohydrate, and $8 \%$ fat $(\mathrm{w} / \mathrm{w})]$. Rats were deprived of food but not water $18 \mathrm{~h}$ prior to administration of the test substances.

Initial toxicological evaluation was carried out by feeding Sprague Dawley rats with tissue cultured Echinacea pupurea adventitious root powder at a dose of $5000 \mathrm{mg} \cdot \mathrm{kg}^{-1}$ diet in two group of five males and five females and animals were observed on daily basis for reversibility, persistence, or delayed occurrence of toxic effects. Gain in body weight was measured on day $0,3,7$, and 14 and at the end of experiment, necropsy analysis was carried with respect to abdominal cavity, adrenal, brain, cranial cavity, oesophagus, heart, intestine, kidney, liver, lung, lymph node, mammary glands, ovary, pancreas, pituitary, prostrate, salivary gland, seminal vesicle, skin, spleen, stomach, testis, thoracic cavity, thymus, thyroid, trachea, urinary bladder, uterus and vagina.

In another set of experiments, Sprague Dawley rats were categorized into four groups of five male and five females each respectively and treated orally with the tissue cultured Echinacea purpurea adventitious root powder at doses ranging from $0,500,1000$ and $2000 \mathrm{mg} \cdot \mathrm{kg}^{-1}$ for a period of 27 days. Food consumption by each group of experimental rats was recorded on day $0,1,2,3$ and 4; and change 
in body weight was recorded on day, $0,3,7,10,14,17$, 21, 24, and 27. Blood samples were collected from a common carotid into heparinized and dry non-heparinized centrifuge tubes. The heparinized blood was used for hematological studies like total erythrocyte count (RBC), hemoglobin concentration (HGB), hematocrit (HCT), mean value of RBC (MCV), mean cell hemoglobin (MCH), mean cell hemoglobin concentration (MCHC), white blood cells (WBC) and platelet (PLT). The serum was separated from the non-heparinized blood and was assayed for glucose (Glu), blood urea nitrogen (BUN), creatinine (Crea), total protein (TP), albumin (Alb), total cholesterol (T-Chol), alanine amino-transferase (ALT), aspartate amino-transferase (AST), alkaline phosphatase (ALP) and albumin and globulin ratio (A/G ratio). Following blood collection, animals were sacrificed for tissue studies. The body weight, absolute organ weight and relative organ weights ( $\mathrm{g} \cdot 100 \mathrm{~g}^{-1}$ body weight) of organs such as brain, heart, liver, spleen and kidney [left (Lt.), right (Rt.)] were assessed. Necropsy analysis was carried with respect to abdominal cavity, adrenal, brain, cranial cavity, oesophagus, heart, intestine, kidney, liver, lung, lymph node, mammary glands, ovary, pancreas, pituitary, prostrate, salivary gland, seminal vesicle, skin, spleen, stomach, testis, thoracic cavity, thymus, thyroid, trachea, urinary bladder, uterus and vagina.

\section{Statistical Analysis}

The results are expressed as mean values with standard deviation. The data obtained from the mutagenicity tests were statistically analyzed using student's t-test. The student's t-test was used to analyze the number of PCE cells among the erythrocytes. One-way parametric ANOVA with Dunnett's test was used to examine the organ weight, body weight, hematological and blood chemistry data. Fisher's exact test was used to evaluate the necropsy data.

\section{Results}

\section{Mutagenicity Test}

The reverse mutation tests using S. typhimurium TA98, TA100, TA1535, and TA1537 and E. coli WP2uvrA showed that the base-pair substitution and frame-shift type of mutations were comparable to the control levels irrespective of dose variation treatments (Tables 2 and 3). There was a slight increase in base-pair mutations with an increase in dose levels ( 2500 and $5000 \mu \mathrm{g} /$ plate); however, these values $(131 \pm 14$ and $128 \pm 18)$ were much lower than the number of mutations observed in the positive control $(411 \pm 18)$.

The in vitro chromosomal aberration test using CHL/IU

Table 2. In vitro reverse mutation test of tissue cultured Echinacea purpurea adventitious roots powder treated $S$. typhimurium and $E$. coli without S-9 mix.

\begin{tabular}{|c|c|c|c|c|c|}
\hline \multirow{3}{*}{$\begin{array}{l}\text { Dose } \\
(\mu \mathrm{g} / \text { plate })\end{array}$} & \multicolumn{5}{|c|}{ Number of revertants/plate ${ }^{\mathrm{z}}$} \\
\hline & \multicolumn{3}{|c|}{ Base-pair substitution type } & \multicolumn{2}{|c|}{ Frame-shift type } \\
\hline & TA100 & TA1535 & WP2uvrA (pKM101) & TA98 & TA1537 \\
\hline 0 & $109 \pm 12$ & $19 \pm 3$ & $108 \pm 8$ & $27 \pm 7$ & $10 \pm 4$ \\
\hline 312.5 & $106 \pm 6$ & $18 \pm 4$ & $113 \pm 11$ & $28 \pm 9$ & $9 \pm 1$ \\
\hline 625 & $99 \pm 7$ & $15 \pm 3$ & $118 \pm 10$ & $24 \pm 4$ & $12 \pm 1$ \\
\hline 1,250 & $122 \pm 7$ & $10 \pm 1$ & $120 \pm 4$ & $24 \pm 2$ & $10 \pm 2$ \\
\hline 2,500 & $131 \pm 14$ & $12 \pm 3$ & $116 \pm 6$ & $23 \pm 1$ & $11 \pm 5$ \\
\hline 5,000 & $128 \pm 18$ & $16 \pm 3$ & $116 \pm 6$ & $26 \pm 7$ & $8 \pm 0$ \\
\hline Positive control & $411 \pm 18$ & $410 \pm 17$ & $423 \pm 16$ & $531 \pm 46$ & $447 \pm 9$ \\
\hline \multicolumn{2}{|l|}{ Strain } & \multicolumn{2}{|c|}{ Positive control } & \multicolumn{2}{|c|}{ Concentration ( $\mu \mathrm{g} /$ plate) } \\
\hline TA100 & & \multicolumn{2}{|c|}{ Sodium azide (SA) } & \multicolumn{2}{|c|}{1.5} \\
\hline TA1535 & & \multicolumn{2}{|c|}{ Sodium azide (SA) } & \multicolumn{2}{|c|}{1.5} \\
\hline WPuvrA (Pkm101) & & \multicolumn{2}{|c|}{ 4-Nitroquinoline 1-oxide (4-NAO) } & \multicolumn{2}{|c|}{5.0} \\
\hline TA98 & & \multicolumn{2}{|c|}{ 2-Nitrofluorene (2-NF) } & \multicolumn{2}{|c|}{5.0} \\
\hline TA1537 & & \multicolumn{2}{|c|}{ 9-Aminoacridine (9-AA) } & \multicolumn{2}{|c|}{80.0} \\
\hline
\end{tabular}

${ }^{\mathrm{z}}$ Mean $\pm \mathrm{SD}, \mathrm{n}=3$. 
Table 3. In vitro reverse mutation test of tissue cultured Echinacea purpurea adventitious roots powder treated S. typhimurium and E. coli with S-9 mix.

\begin{tabular}{|c|c|c|c|c|c|}
\hline \multirow{3}{*}{$\begin{array}{l}\text { Dose } \\
(\mu \mathrm{g} / \text { plate })\end{array}$} & \multicolumn{5}{|c|}{ Number of revertants/plate } \\
\hline & \multicolumn{3}{|c|}{ Base-pair substitution type } & \multicolumn{2}{|c|}{ Frame-shift type } \\
\hline & TA100 & TA1535 & WP2uvrA (pKM101) & TA98 & TA1537 \\
\hline 0 & $122 \pm 17$ & $16 \pm 2$ & $107 \pm 4$ & $26 \pm 9$ & $12 \pm 1$ \\
\hline 312.5 & $118 \pm 13$ & $12 \pm 3$ & $106 \pm 6$ & $27 \pm 3$ & $12 \pm 4$ \\
\hline 625 & $126 \pm 12$ & $14 \pm 1$ & $101 \pm 3$ & $28 \pm 5$ & $13 \pm 3$ \\
\hline 1,250 & $118 \pm 5$ & $13 \pm 2$ & $113 \pm 17$ & $28 \pm 6$ & $11 \pm 3$ \\
\hline 2,500 & $123 \pm 8$ & $16 \pm 4$ & $114 \pm 5$ & $21 \pm 9$ & $12 \pm 4$ \\
\hline 5,000 & $111 \pm 12$ & $13 \pm 2$ & $107 \pm 4$ & $27 \pm 3$ & $14 \pm 2$ \\
\hline Positive control & $473 \pm 12$ & $183 \pm 18$ & $527 \pm 20$ & $422 \pm 25$ & $123 \pm 11$ \\
\hline \multicolumn{2}{|l|}{ Strain } & \multicolumn{2}{|c|}{ Positive control } & \multicolumn{2}{|c|}{ Concentration ( $\mu \mathrm{g} /$ plate $)$} \\
\hline \multicolumn{2}{|l|}{ TA100 } & \multicolumn{2}{|c|}{ 2-Aminoanthracane (2-AA) } & \multicolumn{2}{|c|}{1.0} \\
\hline \multicolumn{2}{|l|}{ TA1535 } & \multicolumn{2}{|c|}{ 2-Aminoanthracane (2-AA) } & \multicolumn{2}{|c|}{2.0} \\
\hline \multicolumn{2}{|l|}{ WPuvrA (Pkm101) } & \multicolumn{2}{|c|}{ 2-Aminoanthracane (2-AA) } & \multicolumn{2}{|c|}{2.0} \\
\hline \multicolumn{2}{|l|}{ TA98 } & \multicolumn{2}{|c|}{ 2-Aminoanthracane (2-AA) } & \multicolumn{2}{|c|}{1.0} \\
\hline \multicolumn{2}{|l|}{ TA1537 } & \multicolumn{2}{|c|}{ 2-Aminoanthracane (2-AA) } & \multicolumn{2}{|c|}{2.0} \\
\hline
\end{tabular}

${ }^{\mathrm{z}}$ Mean $\pm \mathrm{SD}, \mathrm{n}=3$.

mammalian cells did not reveal any abnormalities associated with the TCEPAR powder at doses ranging from 275 to $1100 \mu \mathrm{g} \cdot \mathrm{mL}^{-1}$ (Table 4). However, the tested mutagenic agents mitomycin $\mathrm{C}$ and benzo(a)pyrene (the positive controls) induced significantly frequent chromosomal aberrations.

\section{Toxicological Studies in Experimental Sprague-Dawley Rats}

Single-dose toxicityanalysis: The experimental rats that were fed a single dose of $5000 \mathrm{mg} \cdot \mathrm{kg}^{-1}$ TCEPAR powder showed no signs of toxicity during the experimental period. Both male and female rats showed a gradual gain in body weight during the experimental period (data not shown). There were no observable toxicological symptoms in the external morphological features or in the internal organs, including the abdominal cavity, adrenal glands, brain, cranial cavity, esophagus, heart, intestines, kidneys, liver, lungs, lymph nodes, mammary glands, ovaries, pancreas, pituitary glands, prostate gland, salivary glands, seminal vesicles, skin, spleen, stomach, testis, thoracic cavity, thymus, thyroid, trachea, urinary bladder, uterus, and vagina (data not shown).

Repeated-dose toxicityanalysis: The experimental rats that were fed with 500, 1000, and $2000 \mathrm{mg} \cdot \mathrm{kg}^{-1}$ TCEPAR powder along with their diet over a period of twenty-seven days did not show any signs of toxicity. Both the male and female rats had normal food intake over the observation period of four weeks (Table 5). There was a concurrent gain in body weight with all the tested doses $\left(500-2000 \mathrm{mg} \cdot \mathrm{kg}^{-1}\right)$ in both male and female rats (Fig. 1). Values for hematological parameters such as RBC, HGB, HCT, MCV, MCH, MCHC, WBC count, and PLT count) were all in the control range irrespective of the dose $\left(500,1000\right.$, or $\left.2000 \mathrm{mg} \cdot \mathrm{kg}^{-1}\right)$ of TCEPAR powder (Table 6). Blood chemistry values of the male and female rats are shown in the Table 7. ALT, AST, ALP, Glu, BUN, Crea, T-Chol, TP, Alb, and A/G ratio values in both male and female groups and in the varied dosage treatments of Sprague-Dawley rats were in the normal range, and did not show significant variations (Table 8). As shown in Tables 8 and 9, the absolute and relative body weights, as well as organ weights of the brain, heart, liver, spleen, and kidney of TCEPAR powder-treated male and female rats did not differ from the corresponding values obtained for the control groups. Necropsy examinations of the abdominal cavity, adrenal glands, brain, cranial cavity, esophagus, heart, intestines, kidney, liver, lungs, lymph nodes, mammary glands, ovaries, pancreas, pituitary glands, prostate gland, salivary glands, seminal vesicles, skin, spleen, stomach, testis, thoracic cavity, thymus, thyroid, trachea, urinary bladder, uterus, and vagina revealed no malformation or variation (data not shown). 
Table 4. In vitro chromosome aberration test in CHL/IU cells treated with tissue cultured Echinacea purpurea adventitious roots powder.

\begin{tabular}{|c|c|c|c|c|c|}
\hline S9 mix/time & Test item ${ }^{z}$ & $\begin{array}{c}\text { Dose } \\
\left(\mu \mathrm{g} \cdot \mathrm{mL}^{-1}\right)\end{array}$ & $\begin{array}{l}\text { No. of } \\
\text { cell scored }\end{array}$ & $\begin{array}{l}\text { Percentage of cells } \\
\text { involved in chromosomal } \\
\text { aberrations }\end{array}$ & $\begin{array}{l}\text { Chromosome aberration } \\
\text { cells/100 metaphase } \\
\text { cells }(\%)^{\mathrm{y}}\end{array}$ \\
\hline \multirow{10}{*}{$\begin{array}{l}\text { S9 mix }(-) / \\
6+18 \mathrm{~h}\end{array}$} & \multirow[t]{2}{*}{ Water for injection } & \multirow[t]{2}{*}{0} & 100 & 1 & \multirow[t]{2}{*}{$1.5 \pm 0.7$} \\
\hline & & & 100 & 2 & \\
\hline & \multirow{6}{*}{$\begin{array}{l}\text { Tissue cultured } \\
\text { E. purpurea } \\
\text { adventitious } \\
\text { roots extract }\end{array}$} & \multirow[t]{2}{*}{275} & 100 & 2 & \multirow[t]{2}{*}{$1.0 \pm 0.4$} \\
\hline & & & 100 & 0 & \\
\hline & & \multirow[t]{2}{*}{550} & 100 & 0 & \multirow[t]{2}{*}{$2.0 \pm 0.8$} \\
\hline & & & 100 & 4 & \\
\hline & & \multirow[t]{2}{*}{1100} & 100 & 2 & \multirow[t]{2}{*}{$1.0 \pm 0.4$} \\
\hline & & & 100 & 0 & \\
\hline & \multirow[t]{2}{*}{ MMC } & \multirow[t]{2}{*}{0.05} & 100 & 22 & \multirow[t]{2}{*}{$24.5 \pm 3.5$} \\
\hline & & & 100 & 27 & \\
\hline \multirow{10}{*}{$\begin{array}{l}\text { S9 mix }(+) / \\
6+18 \mathrm{~h}\end{array}$} & \multirow[t]{2}{*}{ Water for injection } & \multirow[t]{2}{*}{0} & 100 & 2 & \multirow[t]{2}{*}{$2.5 \pm 0.7$} \\
\hline & & & 100 & 3 & \\
\hline & \multirow{6}{*}{$\begin{array}{l}\text { Tissue cultured } \\
\text { E. purpurea } \\
\text { adventitious } \\
\text { roots extract }\end{array}$} & \multirow[t]{2}{*}{275} & 100 & 1 & \multirow[t]{2}{*}{$1.5 \pm 0.7$} \\
\hline & & & 100 & 2 & \\
\hline & & \multirow[t]{2}{*}{550} & 100 & 3 & \multirow[t]{2}{*}{$3.5 \pm 0.7$} \\
\hline & & & 100 & 4 & \\
\hline & & \multirow[t]{2}{*}{1100} & 100 & 0 & \multirow[t]{2}{*}{$1.0 \pm 0.4$} \\
\hline & & & 100 & 2 & \\
\hline & \multirow[t]{2}{*}{$\mathrm{B}[\mathrm{a}] \mathrm{P}$} & \multirow[t]{2}{*}{20} & 100 & 24 & \multirow[t]{2}{*}{$24.5 \pm 0.7$} \\
\hline & & & 100 & 25 & \\
\hline \multirow{10}{*}{$\begin{array}{l}\text { S9 mix }(-) / \\
24+0 \mathrm{~h}\end{array}$} & \multirow[t]{2}{*}{ Water for injection } & \multirow[t]{2}{*}{0} & 100 & 0 & $1.0 \pm 0.4$ \\
\hline & & & 100 & 2 & \\
\hline & Tissue cultured & 275 & 100 & 0 & $1.5 \pm 1.1$ \\
\hline & $\begin{array}{l}\text { E. purpurea } \\
\text { adventitious }\end{array}$ & & 100 & 3 & \\
\hline & roots extract & 550 & 100 & 0 & $0.5 \pm 0.7$ \\
\hline & & & 100 & 1 & \\
\hline & & 1100 & 100 & 3 & $1.5 \pm 1.1$ \\
\hline & & & 100 & 0 & \\
\hline & MMC & 0.05 & 100 & 27 & $26.5 \pm 0.7$ \\
\hline & & & 100 & 26 & \\
\hline
\end{tabular}

${ }^{\mathrm{z}} \mathrm{MMC}$, Mictomycin C; B[a]P, Benzo[a]pyrene.

${ }^{\mathrm{y}}$ Chromosomal aberrations observed include chromosomal break, chromatic exchange, chromosome exchange, chromosomal fragments, polyploidy (mean $\pm \mathrm{SD}, \mathrm{n}=3$ ).

\section{Discussion}

Comparative biosafety evaluations of TCEPAR powder and in vitro mutagenicity testing using various strains of S. typhimurium (TA98, TA100, TA1535, and TA1537) and
E. coli (WP2uvrA) did not reveal any significant revertants (base-substitution and frame-shift mutations) compared to controls. Mutagenesis can occur spontaneously or may be induced by a physical, chemical, or biological agent. A previous study has indicated that free radicals can damage 
Table 5. Toxicological studies of tissue cultured adventitious roots of Echinacea purpurea: Food consumption of 4-week dose range studies in Sprague Dawley rats.

\begin{tabular}{lccccc}
\hline $\begin{array}{l}\text { Group/dose } \\
\left(\mathrm{mg} \cdot \mathrm{kg}^{-1}\right)\end{array}$ & 0 & \multicolumn{5}{c}{ Food consumption in g/animal/day (week) } \\
\cline { 2 - 6 } Male & & 1 & 2 & 3 & 4 \\
G1/0 & $23.90 \pm 1.60^{\mathrm{z}}$ & $27.15 \pm 2.54$ & $29.00 \pm 4.14$ & $28.45 \pm 3.31$ & $28.39 \pm 2.38$ \\
G2/500 & $22.84 \pm 1.90$ & $25.77 \pm 2.95$ & $27.00 \pm 0.85$ & $27.31 \pm 3.07$ & $27.08 \pm 0.69$ \\
G3/1000 & $23.97 \pm 2.68$ & $28.22 \pm 3.43$ & $29.34 \pm 3.67$ & $29.31 \pm 3.18$ & $29.73 \pm 3.53$ \\
G4/2000 & $23.32 \pm 1.64$ & $27.02 \pm 2.43$ & $28.91 \pm 1.15$ & $28.96 \pm 3.61$ & $27.52 \pm 2.91$ \\
Female & & & & $18.75 \pm 3.68$ & $21.44 \pm 2.15$ \\
G1/0 & $17.07 \pm 1.53$ & $18.68 \pm 1.26$ & $19.88 \pm 1.36$ & $21.66 \pm 1.74$ & $20.72 \pm 3.37$ \\
G2/500 & $16.86 \pm 0.92$ & $18.28 \pm 1.30$ & $21.60 \pm 2.29$ & $18.09 \pm 2.43$ \\
G3/1000 & $17.57 \pm 2.32$ & $18.56 \pm 0.88$ & $18.77 \pm 1.87$ & $21.53 \pm 1.63$ \\
G4/2000 & $18.77 \pm 0.89$ & $19.55 \pm 0.67$ & $20.91 \pm 2.35$ & $21.18 \pm 1.43$ \\
\hline
\end{tabular}

${ }_{\mathrm{z}}=5$, values are mean \pm SD.
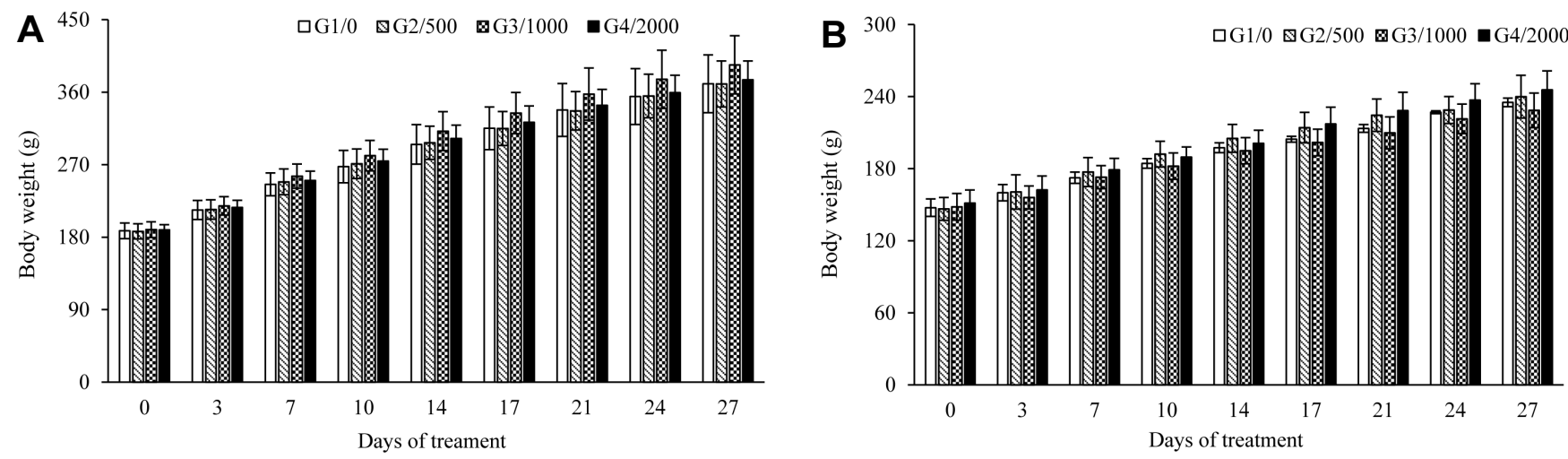

Fig. 1. Toxicological studies of tissue cultured adventitious roots of Echinacea purpurea: Changes in body weight of 4-week dose range studies in Sprague Dawley rats with repeated oral dose toxicity study. (A) male, (B) female. (group/dose, mg $\mathrm{kg}^{-1}$ ).

Table 6. Toxicological studies of tissue cultured adventitious roots of Echinacea purpurea on Sprague Dawley rats: Hematological parameters ${ }^{\mathrm{z}}$.

\begin{tabular}{|c|c|c|c|c|c|c|c|c|}
\hline $\begin{array}{l}\text { Group/dose } \\
\left(\mathrm{mg} \cdot \mathrm{kg}^{-1}\right)\end{array}$ & $\begin{array}{c}\mathrm{RBC} \\
\left(\times 10^{6} \text { cells } / \mu \mathrm{L}\right)\end{array}$ & $\begin{array}{c}\mathrm{HGB} \\
(\mathrm{g} / \mathrm{dl})\end{array}$ & $\begin{array}{l}\text { HCT } \\
(\%)\end{array}$ & $\begin{array}{l}\text { MCV } \\
\text { (fl) }\end{array}$ & $\begin{array}{l}\mathrm{MCH} \\
(\mathrm{pg})\end{array}$ & $\begin{array}{l}\mathrm{MCHC} \\
\text { (g/dl) }\end{array}$ & $\begin{array}{c}\text { WBC } \\
\left(\times 10^{3} \text { cells } / \mu \mathrm{L}\right)\end{array}$ & $\begin{array}{c}\text { PLT } \\
\left(\times 10^{3} \text { cells } / \mu \mathrm{L}\right)\end{array}$ \\
\hline \multicolumn{9}{|l|}{ Male } \\
\hline G1/0 & $7.70 \pm 0.03^{y}$ & $15.9 \pm 0.5$ & $44.5 \pm 1.3$ & $57.7 \pm 1.7$ & $20.5 \pm 0.7$ & $35.7 \pm 0.3$ & $8.56 \pm 1.89$ & $1,247 \pm 154$ \\
\hline G2/500 & $7.88 \pm 0.22$ & $15.8 \pm 0.4$ & $44.1 \pm 0.4$ & $55.9 \pm 1.7$ & $20.0 \pm 0.6$ & $35.9 \pm 0.5$ & $7.53 \pm 1.41$ & $1,216 \pm 143$ \\
\hline G3/1000 & $7.67 \pm 0.54$ & $15.4 \pm 0.6$ & $43.3 \pm 1.6$ & $56.6 \pm 2.3$ & $20.2 \pm 0.7$ & $35.7 \pm 0.3$ & $7.96 \pm 1.64$ & $1,238 \pm 113$ \\
\hline G4/2000 & $7.83 \pm 0.29$ & $16.2 \pm 0.4$ & $45.0 \pm 1.2$ & $57.6 \pm 1.8$ & $20.7 \pm 0.7$ & $35.9 \pm 0.2$ & $7.73 \pm 1.76$ & $1,240 \pm 105$ \\
\hline \multicolumn{9}{|l|}{ Female } \\
\hline G1/0 & $7.68 \pm 0.21$ & $15.4 \pm 0.4$ & $42.8 \pm 1.1$ & $55.8 \pm 0.9$ & $20.0 \pm 0.4$ & $35.9 \pm 0.6$ & $4.53 \pm 0.76$ & $1,270 \pm 28$ \\
\hline G2/500 & $7.82 \pm 0.42$ & $15.6 \pm 0.5$ & $43.5 \pm 1.2$ & $55.8 \pm 1.9$ & $20.0 \pm 0.6$ & $35.8 \pm 0.2$ & $6.31 \pm 1.63$ & $1,166 \pm 93$ \\
\hline G3/1000 & $7.58 \pm 0.32$ & $15.5 \pm 0.3$ & $42.5 \pm 0.9$ & $56.7 \pm 1.5$ & $20.5 \pm 0.6$ & $36.5 \pm 0.4$ & $5.69 \pm 2.05$ & $1,300 \pm 56$ \\
\hline G4/2000 & $7.75 \pm 0.35$ & $15.4 \pm 0.5$ & $42.6 \pm 1.4$ & $55.0 \pm 1.8$ & $19.9 \pm 0.9$ & $36.3 \pm 0.6$ & $6.05 \pm 1.47$ & $1,332 \pm 106$ \\
\hline
\end{tabular}

${ }^{\mathrm{z}}$ Findings of 4 -week dose range test.

${ }^{\mathrm{y}} \mathrm{n}=5$, values are mean \pm SD. 
Table 7. Toxicological studies of tissue cultured adventitious roots of Echinacea purpurea on Sprague Dawley rats: Blood chemistry.

\begin{tabular}{|c|c|c|c|c|c|c|c|c|c|c|}
\hline $\begin{array}{l}\text { Group/dose } \\
\left(\mathrm{mg} \cdot \mathrm{kg}^{-1}\right)\end{array}$ & $\begin{array}{l}\text { ALT } \\
(\mathrm{U} / \mathrm{I})\end{array}$ & $\begin{array}{l}\text { AST } \\
(\mathrm{U} / \mathrm{l})\end{array}$ & $\begin{array}{l}\text { ALP } \\
(\mathrm{U} / \mathrm{I})\end{array}$ & $\begin{array}{c}\text { Glu } \\
\text { (mg/dL) }\end{array}$ & $\begin{array}{c}\text { BUN } \\
\text { (mg/dL) }\end{array}$ & $\begin{array}{c}\text { Crea } \\
\text { (mg/dL) }\end{array}$ & $\begin{array}{l}\text { T-Chol } \\
\text { (mg/dL) }\end{array}$ & $\begin{array}{c}\mathrm{TP} \\
(\mathrm{g} / \mathrm{dL})\end{array}$ & $\begin{array}{c}\text { Alb } \\
\text { (g/dL) }\end{array}$ & $\begin{array}{l}\mathrm{A} / \mathrm{G} \\
\text { ratio }\end{array}$ \\
\hline \multicolumn{11}{|l|}{ Male } \\
\hline $\mathrm{G} 1 / 0$ & $32.8 \pm 3.7^{y}$ & $130.5 \pm 33.6$ & $425.6 \pm 42.8$ & $118.47 \pm 15.26$ & $13.58 \pm 1.34$ & $0.39 \pm 0.11$ & $81.47 \pm 9.27$ & $5.9 \pm 0.3$ & $2.5 \pm 0.1$ & $0.75 \pm 0.08$ \\
\hline G2/500 & $29.4 \pm 3.0$ & $130.1 \pm 35.6$ & $428.1 \pm 71.3$ & $111.64 \pm 10.31$ & $12.77 \pm 1.29$ & $0.46 \pm 0.08$ & $61.81 \pm 11.87$ & $5.8 \pm 0.1$ & $2.4 \pm 0.0$ & $0.70 \pm 0.04$ \\
\hline G3/1000 & $30.0 \pm 4.9$ & $154.5 \pm 13.4$ & $400.2 \pm 51.2$ & $115.08 \pm 8.02$ & $13.15 \pm 0.59$ & $0.41 \pm 0.03$ & $79.41 \pm 13.58$ & $6.0 \pm 0.1$ & $2.5 \pm 0.0$ & $0.70 \pm 0.04$ \\
\hline G4/2000 & $28.5 \pm 3.4$ & $165.9 \pm 18.2$ & $448.3 \pm 70.8$ & $114.7 \pm 4.25$ & $14.18 \pm 1.39$ & $0.47 \pm 0.13$ & $68.79 \pm 15.94$ & $5.9 \pm 0.1$ & $2.4 \pm 0.1$ & $0.69 \pm 0.03$ \\
\hline \multicolumn{11}{|l|}{ Female } \\
\hline $\mathrm{G} 1 / 0$ & $27.3 \pm 3.1$ & $146.3 \pm 15.3$ & $281.7 \pm 42.3$ & $107.94 \pm 9.09$ & $15.72 \pm 2.78$ & $0.57 \pm 0.03$ & $77.78 \pm 11.24$ & $6.5 \pm 0.3$ & $2.9 \pm 0.2$ & $0.82 \pm 0.07$ \\
\hline G2/500 & $25.3 \pm 3.0$ & $134.9 \pm 38.5$ & $260.2 \pm 33.7$ & $111.27 \pm 14.8$ & $15.21 \pm 2.22$ & $0.44 \pm 0.10$ & $103.27 \pm 0.10$ & $6.6 \pm 0.4$ & $3.0 \pm 0.2$ & $0.83 \pm 0.06$ \\
\hline G3/1000 & $26.4 \pm 2.2$ & $131.3 \pm 33.2$ & $303.1 \pm 88.9$ & $112.48 \pm 8.98$ & $15.73 \pm 1.71$ & $0.47 \pm 0.07$ & $81.15 \pm 11.24$ & $6.2 \pm 0.3$ & $2.7 \pm 0.2$ & $0.79 \pm 0.07$ \\
\hline G4/2000 & $26.9 \pm 3.0$ & $131.8 \pm 45.6$ & $276.4 \pm 43.1$ & $104.27 \pm 9.34$ & $17.04 \pm 3.62$ & $0.52 \pm 0.14$ & $71.83 \pm 21.50$ & $6.3 \pm 0.3$ & $2.9 \pm 0.1$ & $0.85 \pm 0.09$ \\
\hline
\end{tabular}

${ }^{\mathrm{z}}$ Findings of 4 -week dose range test.

${ }^{y_{n}}=5$, values are mean \pm SD.

Table 8. Toxicological studies of tissue cultured adventitious roots of Echinacea purpurea on Sprague Dawley rats: Absolute organ weight ${ }^{\mathrm{z}}$.

\begin{tabular}{lccccccc}
\hline \multirow{7}{*}{$\begin{array}{l}\text { Group/dose } \\
\left(\mathrm{mg} \cdot \mathrm{kg}^{-1}\right)\end{array}$} & B.W. & Brain & Heart & Liver & Spleen & Kidney \\
\cline { 2 - 8 } & & & & & & Lt. & Rt \\
\hline Male & & & & & & & \\
G1/0 & $344.86 \pm 31.93^{y}$ & $1.81 \pm 0.06$ & $1.17 \pm 0.05$ & $9.79 \pm 1.25$ & $0.69 \pm 0.10$ & $1.16 \pm 0.09$ & $1.18 \pm 0.09$ \\
G2/500 & $344.38 \pm 29.07$ & $1.89 \pm 0.11$ & $1.14 \pm 0.09$ & $9.31 \pm 0.66$ & $0.74 \pm 0.10$ & $1.20 \pm 0.11$ & $1.22 \pm 0.66$ \\
G3/1000 & $363.24 \pm 38.86$ & $1.89 \pm 0.02$ & $1.18 \pm 0.06$ & $10.76 \pm 1.64$ & $0.72 \pm 0.04$ & $1.31 \pm 0.17$ & $1.29 \pm 0.14$ \\
G4/2000 & $350.02 \pm 22.85$ & $1.85 \pm 0.07$ & $1.11 \pm 0.07$ & $10.27 \pm 1.04$ & $0.69 \pm 0.07$ & $1.27 \pm 0.08$ & $125 \pm 0.14$ \\
Female & & & & & & & \\
G1/0 & $215.63 \pm 1.83$ & $1.75 \pm 0.10$ & $0.82 \pm 0.06$ & $5.88 \pm 0.06$ & $0.41 \pm 0.18$ & $0.75 \pm 0.04$ & $0.76 \pm 0.03$ \\
G2/500 & $222.82 \pm 12.83$ & $1.80 \pm 0.11$ & $0.83 \pm 0.05$ & $6.69 \pm 0.75$ & $0.46 \pm 0.08$ & $0.80 \pm 0.07$ & $0.83 \pm 0.06$ \\
G3/1000 & $213.36 \pm 12.04$ & $1.77 \pm 0.12$ & $0.79 \pm 0.05$ & $6.02 \pm 0.36$ & $0.47 \pm 0.05$ & $0.78 \pm 0.09$ & $0.80 \pm 0.07$ \\
G4/2000 & $228.17 \pm 14.57$ & $1.77 \pm 0.09$ & $0.82 \pm 0.02$ & $6.58 \pm 0.60$ & $0.49 \pm 0.04$ & $0.81 \pm 0.08$ & $0.83 \pm 0.09$ \\
\hline
\end{tabular}

${ }^{\mathrm{z}}$ Findings of 4 -week dose range test.

${ }^{\mathrm{y}} \mathrm{n}=5$, values are mean \pm SD.

DNA and cause cytotoxicity and mutagenicity (Zahin et al., 2010). Echinacea extracts have been used for years and they have been reported to be safe for tested microorganisms (i.e., S. typhimurium TA98, TA100, TA1535, TA1537, and TA 1538), mammalian cells, and mice cells as well as tested rats and mice (Mengs et al., 1991). However, a recent study by Caillet et al. (2011) indicated that a commercial product of Echinacea (Echinoforce) exhibited mutagenic activity in the S. typhimurium strain TA1535/ pSK1002, but the authors did not provide a detailed composition profile for the tested Echinacea extract product.
Nevertheless, their result did raise some concern about the safety of Echinacea products and potential hazards that could result from the long-term use of such plant extracts. In the present study, TCEPAR powder was used in the dose range of 312.5-5000 $\mu \mathrm{g} / \mathrm{plate}$, and the mutation frequencies for the tested $S$. typhimurium strains TA98, TA100, TA1535, and TA1537 and E. coli strain WP2uvrA did not change significantly compared to spontaneous mutation frequencies (Tables 2 and 3). Indeed, none of the tested concentrations induced a significant increase in the revertant number of $S$. typhimurium strains TA98, 
Table 9. Toxicological studies of tissue cultured adventitious roots of Echinacea purpurea on Sprague Dawley rats: Relative body weights ${ }^{z}$.

\begin{tabular}{lccccccc}
\hline \multirow{8}{*}{$\begin{array}{l}\text { Group/dose } \\
\left(\mathrm{mg} \cdot \mathrm{kg}^{-1}\right)\end{array}$} & B.W. & Brain & Heart & Liver & Spleen & Kidney \\
\cline { 2 - 8 } & & & & & & Lt. & Rt \\
\hline Male & & & & & & & \\
G1/0 & $344.9 \pm 31.90^{y}$ & $0.5 \pm 0.04$ & $0.3 \pm 0.02$ & $2.8 \pm 0.12$ & $0.2 \pm 0.02$ & $0.34 \pm 0.03$ & $0.34 \pm 0.03$ \\
G2/500 & $344.38 \pm 29.07$ & $0.55 \pm 0.04$ & $0.33 \pm 0.01$ & $2.71 \pm 0.19$ & $0.22 \pm 0.01$ & $0.35 \pm 0.04$ & $0.36 \pm 0.03$ \\
G3/1000 & $363.24 \pm 38.86$ & $0.52 \pm 0.05$ & $0.33 \pm 0.03$ & $2.95 \pm 0.19$ & $0.20 \pm 0.02$ & $0.36 \pm 0.03$ & $0.36 \pm 0.02$ \\
G4/2000 & $350.02 \pm 22.85$ & $0.53 \pm 0.02$ & $0.32 \pm 0.02$ & $2.93 \pm 0.19$ & $0.20 \pm 0.02$ & $0.36 \pm 0.02$ & $0.36 \pm 0.02$ \\
Female & & & & & & & \\
G1/0 & $215.63 \pm 1.83$ & $0.81 \pm 0.05$ & $0.38 \pm 0.03$ & $2.73 \pm 0.09$ & $0.19 \pm 0.02$ & $0.35 \pm 0.02$ & $0.35 \pm 0.01$ \\
G2/500 & $222.82 \pm 12.83$ & $0.81 \pm 0.06$ & $0.37 \pm 0.02$ & $3.00 \pm 0.21$ & $0.21 \pm 0.03$ & $0.36 \pm 0.02$ & $0.37 \pm 0.02$ \\
G3/1000 & $213.36 \pm 12.04$ & $0.83 \pm 0.05$ & $0.37 \pm 0.03$ & $2.82 \pm 0.11$ & $0.22 \pm 0.02$ & $0.36 \pm 0.03$ & $0.37 \pm 0.02$ \\
G4/2000 & $228.17 \pm 14.57$ & $0.78 \pm 0.03$ & $0.36 \pm 0.02$ & $2.88 \pm 0.18$ & $0.21 \pm 0.01$ & $0.36 \pm 0.02$ & $0.37 \pm 0.03$ \\
\hline
\end{tabular}

${ }^{\mathrm{z}}$ Findings of 4 -week dose range test.

${ }^{\mathrm{y}} \mathrm{n}=5$, values are mean \pm SD.

TA100, TA1535, and TA1537 and E. coli strain WP2uvrA with or without the S9 mix. Thus, TCEPARs appear to be non-genotoxic. These results concur with earlier reported results that Echinacea purpurea is safe, with little toxicity and mutagenicity (Tsai et al., 2012).

Studies of possible chromosomal aberrations caused by TCEPAR powder at the range $275-1100 \mu \mathrm{g} / \mathrm{mL}$ conducted using CHL/IU cells with and without the S9 mixture did not show any significant chromosomal abnormalities (Table 4). In contrast, the tested mutagenic agents mitomycin $C$ and benzo(a)pyrene (the positive controls) induced significantly frequent chromosomal aberrations including chromosomal breaks, chromatic exchanges, chromosome exchanges, chromosomal fragments, and polyploidy. The major ingredients of Echinacea purpurea adventitious roots are phenolics, especially caffeic acid derivatives (namely, cichoric acid, caftaric acid, chlorogenic acid, echinacoside, and cynarin) (Paek et al., 2009; Wu et al., 2007). In general, phenolics and specifically caffeic acid derivatives have high antioxidant and antimutagenic activities (Paek et al., 2009; Tsai et al., 2012). TCEPARs have been reported to possess higher concentrations of phenolics and flavonoids (Jeong et al., 2009; Wu et al., 2008) Some studies support the view that compounds with antioxidant activity can inhibit mutations because they scavenge free radicals or induce antioxidant enzyme activity (Yen and Chen, 1995).

Four weeks of repeated toxicity tests in Sprague-Dawley rats did not show death or even reversibility, persistence, or delayed toxic effects (irrespective of dose levels administered). Food consumption levels were routine (Table 5), There was a concurrent increase in body weight during the observation period (Fig. 1) in both the male and female groups of experimental animals. Hematological data revealed normality in the observed parameters (Table 6). It was reported that ALT and AST levels increased considerably with treatment of tissue-cultured mountain ginseng adventitious root powder in experimental male rats (Sivakumar et al., 2006), suggesting that the components of tissue-cultured mountain ginseng adventitious root powder directly affect liver and kidney functions. In the present study, no such fluctuations were observed in ALT and AST levels and in varied blood chemistry parameters (Table 7). The absolute and relative organ weights (e.g., of the brain, heart, liver, spleen, and kidney) were in the normal range compared to corresponding weights in the controls (Tables 8 and 9). Furthermore, necropsy examinations revealed no malformation in the abdominal cavity, adrenal glands, brain, cranial cavity, esophagus, heart, intestines, kidney, liver, lungs, lymph nodes, mammary glands, ovaries, pancreas, pituitary glands, prostate gland, salivary glands, seminal vesicles, skin, spleen, stomach, testis, thoracic cavity, thymus, thyroid, trachea, urinary bladder, uterus, and vagina (Data not shown). Mutagenicity tests of Echinacea purpurea powder and extract (obtained from field-grown plants) carried out by Mengs et al. (1991) in microorganisms and mammalian cells in vitro and in mice gave negative results. Single oral 
$\left(2500 \mathrm{mg} \cdot \mathrm{kg}^{-1}\right)$ and intravenous $\left(50 \mathrm{~mL} \cdot \mathrm{kg}^{-1}\right)$ doses of Echinacea purpurea extract proved virtually nontoxic to rats and mice. Mengs et al. (1991) also performed necropsy studies after 4 weeks of oral administration of Echinacea purpurea extract in rats and observed no toxic effects. Perri et al. (2006) did a literature survey on the safety and efficacy of $E$. purpurea during pregnancy and lactation in humans and reported non-teratogenic effects.

In conclusion, the present experimental data from safety and toxicological evaluations support the safety and nontoxicity of Echinacea purpurea. The results indicate that TCEPAR biomass is safe for human consumption and can be used in the pharmaceutical and food industries. The production of the adventitious root biomass in commercial bioreactors can pave the way for year-round production of the raw material in a contamination-free environment.

\section{Literature Cited}

Abbasi, B.H., P.K. Saxena, S.J. Murch, and C.Z. Liu. 2007. Echinacea biotechnology: Challenges and opportunities. In Vitro Cell. Dev. Biol. Plant 43:481-492.

Ames, B., J. McCann, and I.E. Yamasaki. 1975. Methods for detecting carcinogens and mutagens with Salmonella/mammalian microsome mutagenicity test. Mut. Res. 31:347-364.

Bauer, R. and H. Wagner. 1991. Echinacea species as potential immunostimulating drugs, p. 253-321. In: H. Wagner and N.R. Farnsworth (ed.) Economic and medicinal plant research, Vol. 5. Academic press, New York, USA.

Barrett, B. 2003. Medicinal properties of Echinacea: A critical review. Phytomed. 10:66-86.

Blumenthal, M. 2003. The ABC clinical guide to herbs. American Botanical Council, Thieme, New York, USA.

Caillet, S., S. Lessard, G. Lamoureux, and M. Lacroix. 2011. Umu test applied for screening natural antimutagenic agents. Food Chem. 124:229-248.

Jeong, J.A., C.H. Wu, H.N. Murthy, E.J. Hahn, and K.Y. Paek. 2009. Application of airlift bioreactors for the production of adventitious root biomass and caffeic acid derivatives of Echinacea purpurea. Biotechnol. Bioprocess Eng. 14:91-98.

Marriott, B.M. 2003. An introduction to dietary supplements of plant origin, p. 1-17. In: M. Maffi (ed.). Dietary supplements of plant origin, Taylor and Francis, New York, USA.

Mengs, U., C.B. Clare, and J.A. Poilsey. 1991. Toxicity of Echinacea purpurea, acute, subacute, and genotoxicity studies. Drug Res.
41:1076-1081.

Miller, S.C. 2005. Echinacea: A miracle herb against ageing and cancer? Evidence in vivo in mice. Evi. Based Compl. Altern. Med. 2:309-314.

Paek, K.Y., H.N. Murthy, and E.J. Hahn. 2009. Establishment of adventitious root cultures of Echinacea purpurea for the production of caffeic acid derivatives, p. 3-16. In: S.M. Jain and P.K. Saxena (eds.). Methods in molecular biology, protocols for in vitro cultures and secondary metabolite analysis of aromatic and medicinal plants, Vol. 547. Humana Press, New York, USA

Percival, S.S. 2000. Use of Echinacea in medicine. Biochem. Pharmacol. 60:155-158.

Perri, D., J.J. Dugoua, E. Mills, and G. Koren. 2006. Safety and efficacy of Echinacea (Echinacea angustifolia, E. purpurea and E. pallida) during pregnancy and lactation. Can. J. Clin. Pharmacol. 13:262-267.

Sivakumar, G., K.W. Yu, J.S. Lee, J.K. Kang, H.L. Lee, W.J. Kim, and K.Y. Paek. 2006. Tissue cultured mountain ginseng adventitious roots ${ }^{\mathrm{TM}}$ : Safety and toxicity evaluation. Eng. Life Sci. 6:372-383.

Tsai, Y.L., S.Y. Chiou, K.C. Chan, J.M. Sung, and S.D. Lin. 2012. Caffeic acid derivatives, total phenol, antioxidant and antimutagenic activities of Echinacea purpurea flower extracts. LWT - Food Sci. Technol. 46:169-176.

Wills, R.B.H., K. Bone, and M. Morgan. 2000. Herbal products: Active constituents, mode of action and quality control. Nutr. Res. Rev. 13:47-77.

Wu, C.H., H.N. Murthy, E.J. Hahn, H.L. Lee, and K.Y. Paek. 2008. Efficient extraction of caffeic acid derivatives from adventitious roots of Echinacea purpurea. Czech J. Food Sci. 26:254-258.

Wu, C.H., H.N. Murthy, E.J. Hahn, and K.Y. Paek. 2007. Large scale cultivation of adventitious roots of Echinacea purpurea in airlift bioreactors for the production of chichoric acid, chlorogenic acid and caftaric acid. Biotechnol Lett. 29:1179-1182.

Yen, G.C. and H.Y. Chen. 1995. Antioxidant activity of various tea extracts in relation to their antimutagenicity. J. Agr. Food Chem. 43:27-32.

Yu, H.C. and M. Kaarlas. 2004. Popularity, diversity, and quality of Echinacea, p. 29-52. In: S. Miller (ed.). Echinacea, the genus Echinacea. CRC Press, Boca Raton, Florida, USA.

Zahin, M., F. Aquil, and I. Ahmad. 2010. Broad spectrum antimutagenecity activity of antioxidant active fraction of Punica granatum L. peel extracts. Mut. Res. 703:99-107. 